\title{
MULTI-SOURCE BASED ACOUSTIC MODEL FOR SPEECH SYNTHESIS
}

\author{
Jianhua Tao Yongguo Kang \\ National Laboratory of Pattern Recognition \\ Institute of Automation, Chinese Academy of Sciences \\ \{jhtao,ygkang\}@nlpr.ia.ac.cn
}

\begin{abstract}
Traditional source-filter model has obvious limitation for speech synthesis in pitch modification due to the lack of spectrum distortion processing. To solve the problem, the paper analyzes the spectrum features of voice source in various F0 ranges and timbres in detail, and generates Muliti-Source (MS) based on analysis results by classifying the voice source into different types. The model enhances the quality of speech synthesis in various speaking mood.
\end{abstract}

\section{INTRODUCTION}

Speech synthesis has been made a rapid progress in the past several years by using corpus based technology. But the current method is limited a lot towards embedded application and personal style. It lacks abilities on pitch and spectrum modification. Thus, source-filter model is still an attractive solution to solve the problem. But, we all know the quality of traditional source-filter model is not very good, not only it simplifies the voice tract, but also it lacks detailed and variegated source model.

Klatt presented a cascade/parallel formant synthesizer in 1980[2], which is significant for exploring the model of vocal tract of speech synthesis. Kong [18] also made some research on the vocal tract and voice source and has made a lot of achievements. Since the formant model can reflect the characteristic of vocal tract and have intuitionistic physical meaning, this paper explores a new way for improving speech synthesis quality based formant model.

The paper employs a Muliti-Source (MS) model for speech synthesis, which is generated through classifying and reconstructing various voice source with various prosodies and timbres, and can get very good speech synthesis quality through dynamic combination in synthesizing.

The paper is organized as follows. Section 2 describes the method of formant extraction, the construction of inverse filter and the voice source parameter generation; Section 3 analyzes voice source with various phoneme, timbre and prosody. Section 4 generates Muliti-Source acoustic model through clustering method based on above analysis; Finally, experiments and conclusions are provided in Section 5. Experiments have proved this model can obtain very good speech synthesis quality for most of phonemes.

\section{INVERSE FILTER BASED FORMANTS}

\subsection{Formant estimation}

Formant estimation is the first step in the generating of MS model. As we know, the transformation function of a second-order digital resonator can be written as:

$$
H(z)=\frac{1}{\left(1-z_{1} / z\right)\left(1-z_{1}^{*} / z\right)}=\frac{1}{1-\left(z_{1}+z_{1}^{*}\right) z^{-1}+\left(z z_{1}^{*}\right) z^{2}}
$$

Thus the transformation function of vocal tract can be written as:

$$
H(z)=\prod_{i=1}^{k} H_{i}(z)
$$

Where $Z_{i}$ is defined by formant frequency and bandwidth. For formant estimation, methods based on linear prediction analysis (LPC) and cepstrum analysis have received considerable attention. In recent years Welling's method [5] and Inverse-Filter Control(IFC) are presented and these methods have gotten good result in formant estimation. The following is the method used in the paper.

In this method, the short-time power spectrum is decomposed into segments, each of which is modeled by a digital second-order resonator. The segment boundaries are optimized by dynamic programming. An advantage of the method is that an explicit smoothing of the formant frequencies along the time axis does not seem to be necessary.

The whole frequency range is divided into $K$ segments with boundaries $\omega_{0}=$ $0, \ldots, \omega_{k}, \ldots, \omega_{K}=\pi$, thus the corresponding secondorder resonator can be defined as:

$$
A_{k}\left(e^{j \omega}\right)=1-\alpha_{k} e^{j \omega}-\beta_{k} e^{j 2 \omega}
$$




$$
\begin{aligned}
& f_{k}=\arccos \left[-\frac{\alpha_{k}\left(1-\beta_{k}\right)}{4 \beta_{k}}\right] \\
& b_{k}=\log \left(-\beta_{k}\right)
\end{aligned}
$$

Where $\alpha_{k}$ and $\beta_{k}$ are the real-valued prediction coefficients. If these prediction coefficients are given, the formant frequency $f_{k}$ and bandwidth $b_{k}$ can be obtained according to the formula (4) and (5), and the value of the prediction error is given by

$$
E\left(\omega_{k-1}, \omega_{k} \mid \alpha_{k}, \beta_{k}\right)=\frac{1}{\pi} \int_{\omega_{k-1}}^{\omega_{k}}\left|S\left(e^{j \omega}\right)\right|^{2}\left|A_{k}\left(e^{j \omega}\right)\right|^{2} d \omega
$$

The error of all segments is

$$
E=\sum_{k=1}^{K} E_{\min }\left(\omega_{k-1}, \omega_{k}\right)
$$

Formant parameters are then obtained according to the formula (4) and (5), if segment boundaries are given,

\subsection{Source Estimation}

There are lots of methods used for source estimation, such as ARMA analysis method (fujisaki)[8], Sum-ofExponentials method [9], inverse-filtering method (Rothenberg), least squares glottal inverse filtering (D.Y.Wong) and joint estimation of an AR system with a linear input model (P.Milenkovic).

Voice source can be obtained by matching source model with the inverse-filter result, and we use LF model as source model, which is presented by Fant, Liljencrants and Lin, as shown in Fig.1. The parameter Tp denotes the instant of the maximum glottal flow. $\mathrm{T0}$ is the fundamental period. Tc denotes the ending of the return phase. Ta is the effective duration of the return phase. Ta determines the spectral tilt of the glottal source. The increase of intensity U0 will cause the increase of low frequency harmonic components while the decrease of lowest value Ee (shown in fig 1) in will bring the decrease of high frequency harmonic components.
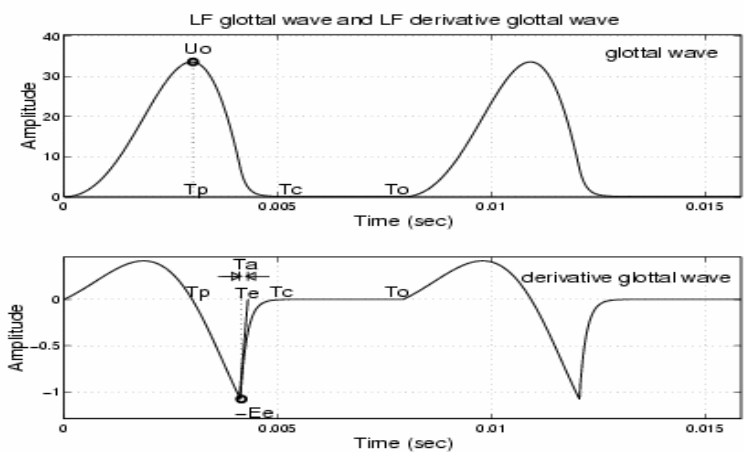

Fig.1 Liljencrants-Fant model

In the paper, the estimation of LF parameter contains two steps: initial parameters estimation and non-linear optimization.
A good initial parametric model is critical for nonlinear optimize and finding the global optimum parameters. It's not difficult to find that Te is one of the easiest acquirable parameters, which can be got from the time when the different voice source signal is minimized. Ee is the value of signal at time Te. Tp can be estimated from the first zero-crossing before Te, and Tc can be estimated from the first point at which the signal value is below a given threshold after Te. As similar to Tc, T0 can be estimated from the first point at which the signal value is below a given threshold before $\mathrm{Tp}$, and the point is limited by open quotient. Ta is the most difficult parameter to be got, and it has been discussed in many papers. In our work, we assumes the following relationships between Ta and Tc, Te,

$$
T_{a}=\frac{2}{3}\left(T_{c}-T_{e}\right)
$$

In our work, there is $\pm 7 \%$ of error distribution in initial parameters, $\pm 4.5 \%$ in Te. Sometimes the error of Ta can be $\pm 20 \%$. In the paper, the non-linear optimized method, which contains dynamic time warping [9], nominalization [10], deciding of dropping points and filter based least squares error, was used to optimize initial parameter estimation. It decreased the error to $\pm 6 \%$, could be used in LF generation.

\section{RELATIONSHIP BETWEEN VOICE SOURCE AND VOCAL TRACT}

\subsection{Analyzing the relationship between voice source and vocal tract}

Traditional source-filter model tries to separate acoustic model into two isolated parts, voice source and vocal tract. It has obvious defect [3]. Lots of experiments have shown that the change of glottal impedance has strong influence between voice source and vocal tract.

Liljencrants proposes Reflection type Line Analog (RTLA) method as the model of voice generation. The method simulates the scatter of air flow in vocal tract, and its digital implement is simple, in addition, losses in the vocal tract can be considered.

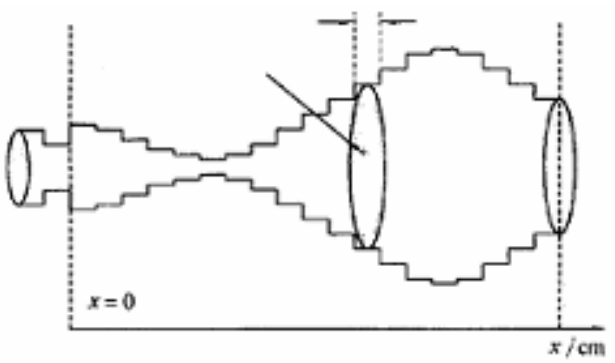

Fig. 2 the model of vocal tract

The vocal tract can be represented as a concatenation of $\mathrm{M}$ lossless acoustic tubes, whose area functions are $\mathrm{A}(\mathrm{i})$ 
and whose length is same, $\mathrm{x} 0$, as depicted in Fig.2. Assuming the pressure and the volume velocity of each tube are $\mathrm{Pi}$ and $\mathrm{Ui}$, respectively, the acoustic impedance can be written:

$$
Z_{i}=\frac{P_{i}}{U_{i}}=\frac{\rho . c}{A(i)}
$$

Because of the change of impedance, the voice source obtained from speech inverse-filter cannot but being related to characteristics of vocal tract. The relation can be described as the following: The glottal flow has the trend of delaying to the ending of the glottal open phase. The shape of air flow leans to right while the glottal opening. And the slope of air flow will be steeper in glottal closing. The non-linear impedance and the scattered excitation in a glottal cycle will cause that instantaneous frequency, phase and formant damping in glottal opening are modulated. Although this interaction is secondary, a filter with this interaction will cause a smooth glottal impulse with various ripple, as depicted in Fig.3(a)(b)(c), which show waveforms of voice sources of vowel "a","i","o" respectively.

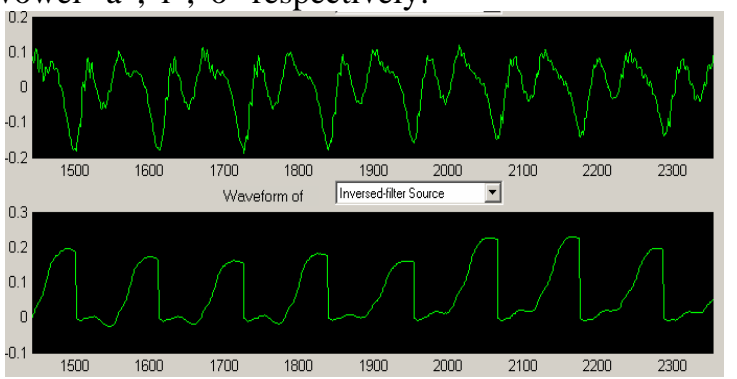

Fig.3(a) voice source of vowel 'a'

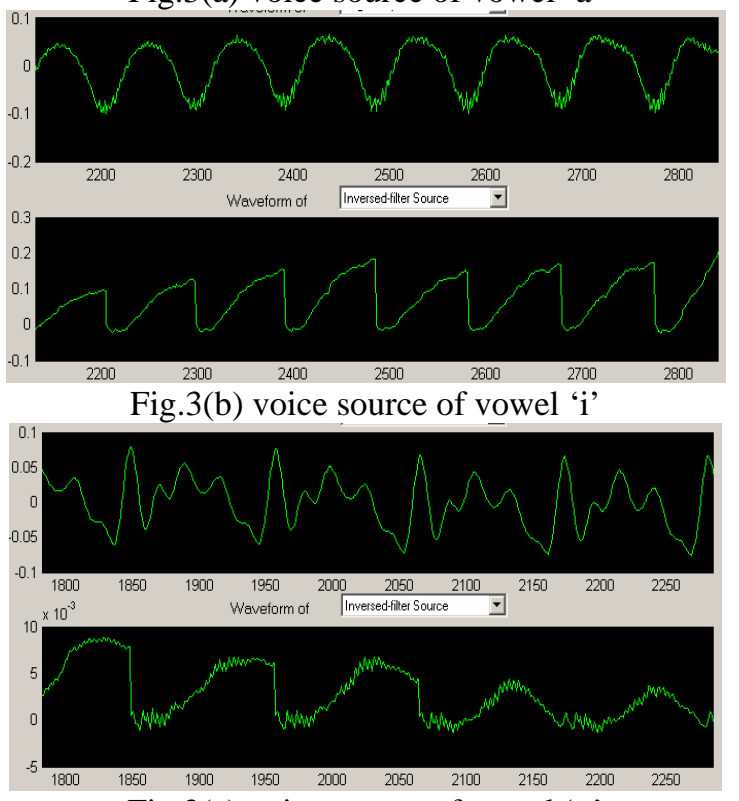

Fig.3(c) voice source of vowel 'o'

To make stable recording style, the corpus used for experiments contains single syllables and phrases, and a few sentences. All of the texts were read 10 times in different prosodic range. To simplify the analysis, we didn't use the unvoiced and plosive voice. Then, we got the some changing rules for U0 and Ee. U0 and Ee will increase with the increasing of vocal pressure (Ptr), while the others keep constant. The value of spectrum, and intensity level (IL) will increase too.

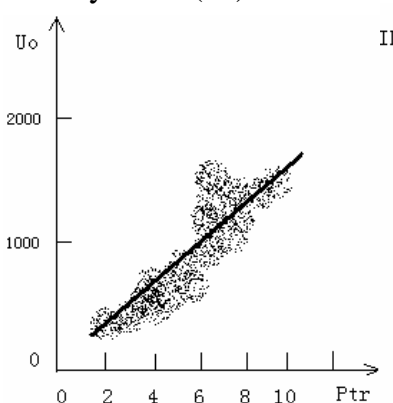

(a)

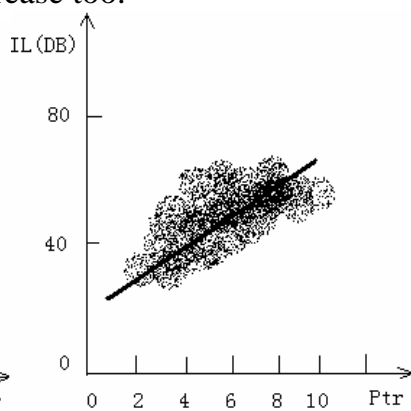

(b)
Fig.4 (a): changes of U0 with Ptr

(b): changes of IL with Ptr

From our experiments, we also could find $\mathrm{Ta} / \mathrm{T} 0$ nearly keep constant for all vowels. U0 in transition of consonants is smaller than that in steady phase, and Ptr in starting point $(5.2 \mathrm{~cm} \mathrm{H} 20)$ of vocal tract is greater than in offset point $(4.1 \mathrm{~cm} \mathrm{H20)}$. The intensity level (IL) of starting point $(63 \mathrm{~dB})$ is greater than ending point $(61 \mathrm{~dB})$ in a glottal cycle.

\subsection{Analysis between voice source and prosodic features}

From Fig 3, voice source shows some scrambling for overlapping formant oscillations of previous glottal cycle. The overlapping arise when the value of F0 is greater. The relationship between Ptr and F0 is less tight than the relationship between Ptr and U0, Ee, IL. Strik and Boves thought the relationship between Ptr and F0 as the result of muscle -movement of larynx [3]. The results could be also confirmed by Fant's work [1].

In our work, we pick up speech units with same phonemes. The duration is similar and the stress feature can be easy distinguished. These units are divided into four groups: unvoiced speech, normal speech, secondary stressed-speech and stressed-speech.

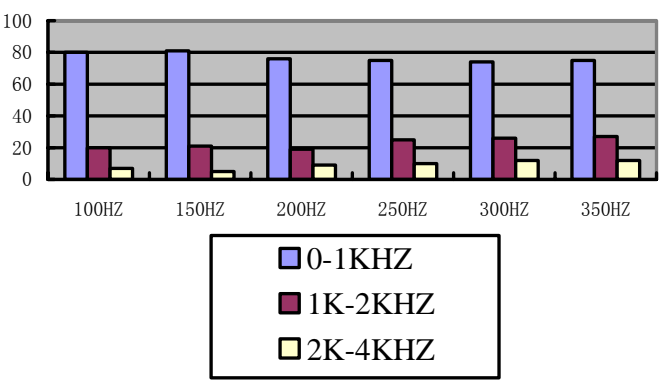


Fig.5 spectral distribution of voice source with various prosodic features

The spectral distribution (0-1000HZ, 1000-2000HZ, 2000-4000HZ) of voice source with different F0s (100HZ, 150HZ, 200HZ, 300HZ, 350HZ) is shown in Fig.5. The distribution is obtained from the mean spectral value in the voiced part of 100 syllables. As shown in the figure, more and more high frequency components appear with the increasing of F0. The feature is a basic reference for the Muliti-Source model generation

\section{MULTI-SOURCE BASED ACOUSTIC MODEL}

As discussed above, there exists impulse fluctuation in voice source due to the interaction between voice source and vocal tract. The feature helps us get more natural and coarticulatory synthesis speech

To syncretize the features in the acoustic model, we classify the source type into various source models in different pitch ranges, and link the source model with formant to generate a Multi-Source Acoustic Model, which synthesizing the speech by using different types of source with specific F0 feature. Fig.6 shows the frame work of model generation and procedure of speech synthesis.

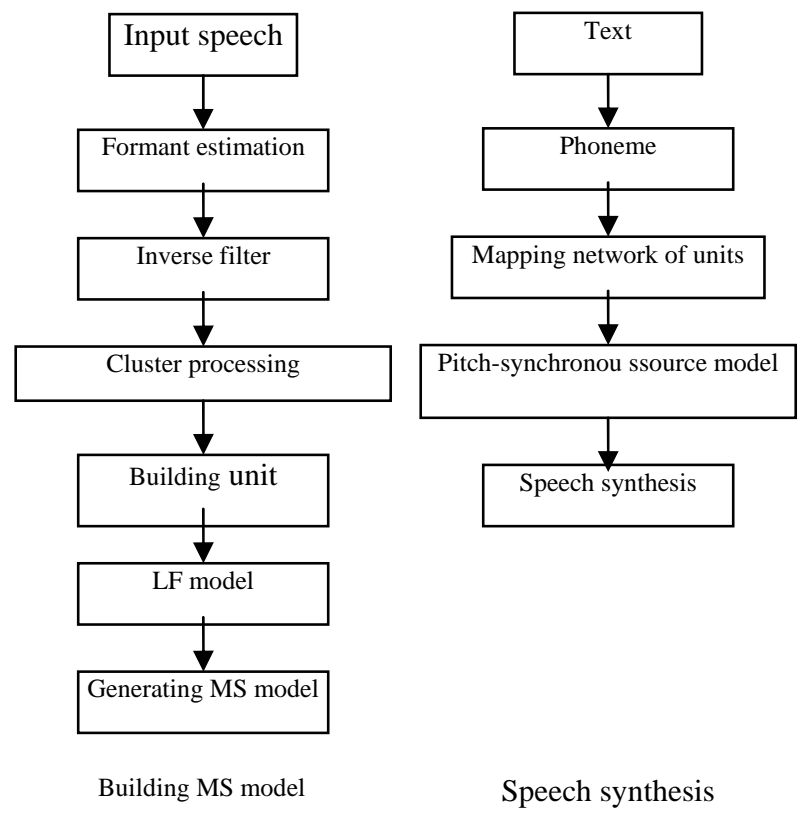

Fig.6 The process of building MS model and speech synthesis

All of the source models are extracted in pitchsynchronous method and are dynamicly-combined while synthesizing. Basic factors of unit-selection in building
Multi-Source model include phoneme p, current f0, duration d, current time t.

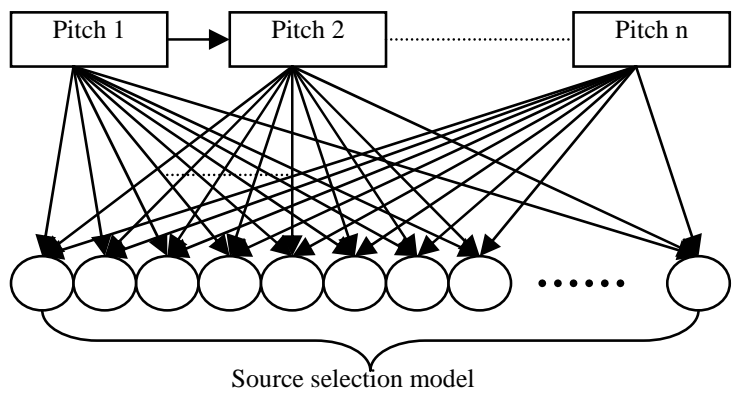

Fig.7 select the source from MS model

In source selection, the cost function is,

$\Delta_{n}=f\left(\vec{X}, \vec{C}_{n}\right)=\vec{A} \bullet\left(\vec{X}-\vec{C}_{n}\right)$

Here, $\Delta_{n}$ is the cost by selecting source $\mathrm{n}$.

$\vec{X}=\left(p, f_{0}, d, t\right)$, are the context information, they are phoneme $\mathrm{p}$, current $\mathrm{f} 0$, the duration $\mathrm{d}$ of phoneme and the central time $t$ of current pitch.

$\vec{C}_{n}=\left(p_{n}^{\prime}, f_{0, n}^{\prime}, d_{n}^{\prime}, t_{n}^{\prime}\right)$, are the context information related to specific source candidate $n$.

From many source candidates, there also exists the transition probability between two time slice during the smoothing of the spectrum. The results improve the final candidate determination. Here, transition probability is defined as the following,

$p_{i, j}=P\left(c_{i}, c_{j}\right)$, where $\mathrm{i}$ and $\mathrm{j}$ indicate the different source candidates. Then, the candidates determination becomes a kind of path searching, and solved by viterbi algorithm.

\section{EXPERIMENTAL RESULTS}

To show the difference among LF model, MS model and PSOLA model, we did an experiment to generate speech synthesis result with different pitch range, from $80 \mathrm{~Hz}$ to $160 \mathrm{~Hz}$.

50 sentence with 15 syllables in each were selected for experiments. Original speech was recorded by a male speaker. MOS test was used, and 5 peoples were asked to evaluate the synthesized speech with 5 levels according to following criteria,

5: very good, without any spectrum distortion

4: good, with a little spectrum distortion

3: fair, can be hear clearly but with some spectrum distortion

2: bad, speaks like a machine, with lots of spectrum distortion, some word cannot be heard clearly

1: very bad, cannot be heard clearly and is not understandable. 


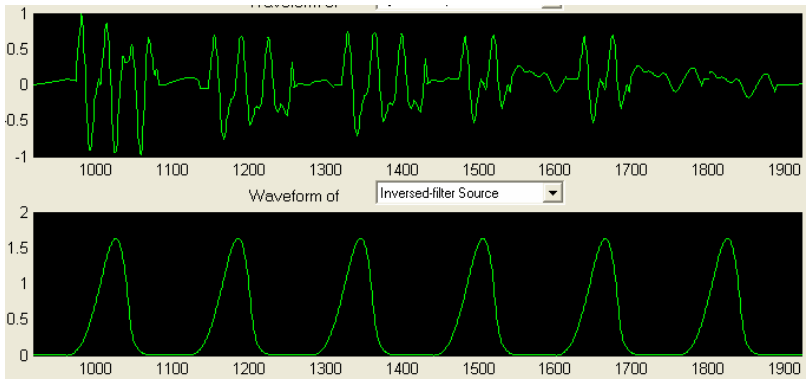

(a) speech synthesis result with LF model

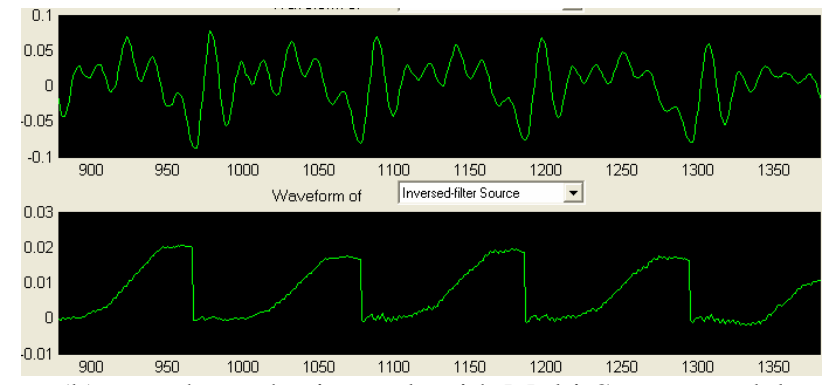

(b) speech synthesis result with Multi-Source model

Fig.8 Speech synthesis results with LF model and MS model

Fig.8 shows the speech synthesis results with LF model and Multi-Source model. The result of MOS test is shown in Fig.9. The testing pitch ranges were separated into four types, $80-180 \mathrm{~Hz}, 150-300 \mathrm{~Hz}, 200-400 \mathrm{~Hz}$ and $250-500 \mathrm{~Hz}$.

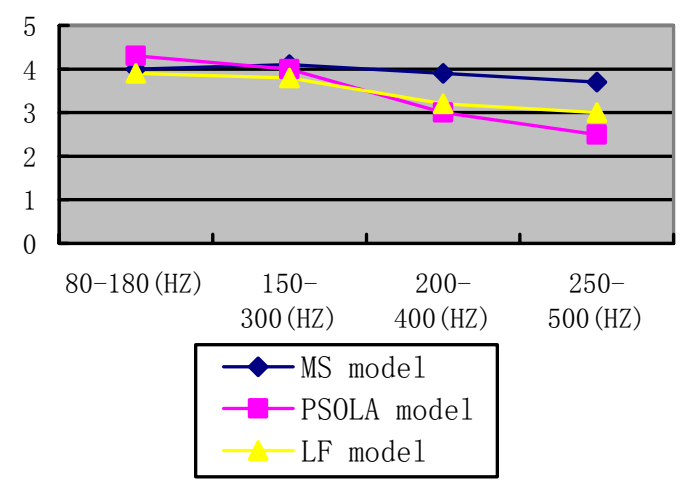

Fig.9 Scores of synthesized speech of various source model with various F0

Due to low frequency character of male speaker, the experiment show there are not too much different among three methods with the F0 range $80 \mathrm{~Hz}-180 \mathrm{~Hz}$. Nearly all of the methods generates good result in low frequency band. But it shows big difference in higher frequency band, where MS model works much better than two other methods. It proves the good quality of MS model.

\section{CONCLUSIONS}

Glottal features have deep influence for voice source during the speech generation. Traditional source-filter model has obvious limitation for speech synthesis in pitch modification. To solve the problem, the paper proposes a Multi-Source model. As we know, personalized style is more and more important for speech synthesis, we could not expect to solve all of the problem by just increasing the corpus. It's still necessary to get a high-quality acoustic model. The paper is based on cascade formant model, detailed features of timbre is not analyzed here. There is still quite a lot of work to do. Future work is be focused on more detailed source classification and more reliable source candidate selection. Creaky voice will also be analyzed later.

\section{REFERENCES}

[1] G. Fant, Gao fen, "Speech science and speech technologies", The commercial press.

[2] Klatt, D.H., "Software for a cascade/ parallel formant synthesizer", Journal of Acoustic Society of America, Vol.67, No.3, pp.971-995.

[3] R.W.Schafer and L. R. Rabiner, System for Automatic Formant Analysis of Voiced Speech, Journal of Acoustic Society of America, Vol.47,No.2.pp. 634-648,1970.

[4] A.Watanabe, Formant Estimation Method Using Inverse-Filter Control, IEEE Trans. On Speech and Audio Processing, Vol.9, No.4, May 2001.

[5] L.Welling ,H.Ney, Formant Estimation for Speech Recognition, IEEE Trans. On Speech and Audio Processing, Vol.6, No.1, January 1998.

[6] D.J.Wong, J.D. Markel, A.H. Gray, Least squares glottal inverse filtering from acoustic speech wave, IEEE Trans. On Acoustics, Speech, and Signal Processing, Vol.ASSP-27, No.4, August 1979.

[7] Rothenberg, M.R., A new inverse-filtering technique for deriving the glottal air flow waveform during voicing, Journal of Acoustic Society of America, pp. 1632-1645.

[8] H. fujisaki, Recent Research towards Advanced ManMachine Interface through Spoken Language, Elsevier Science,1996.

[9] A.K. Krishnamurthy, Glottal Source Estimation Using a Sum-of-Exponentials Model, IEEE Trans. On Signal Processing, Vol. 40, No. 3, March 1992. 
[10] P.Milenkovic, Glottal Inverse Filtering by Joint Estimation of an AR System with a Linear Input Model, IEEE Trans. On Acoustics, Speech, and Signal Processing, Vol.ASSP-34, No.1, February 1986.

[11] M.D. Plumpe, T.F. Quatieri, D.A. Reynolds, Modeling of the Glottal Flow Derivative Waveform with Application to Speaker Identification, IEEE Trans. On Speech and Audio Processing, Vol.7, No.5, September 1999.

[12] Fant, G. \& Lin, Q. , Frequency domain interpretation and derivation of glottal flow parameters. STL-QPSR 2-3, pp. 1-21. 1988

[13] Jansen, J. Automatische extractie van parameters voor het stembron-model van Liljencrants \& Fant. Unplubished doctoral dissertation, Nijmegen. 1990

[14] Klatt, D.H. \& Klatt, L. Analysis, synthesis, and perception of voice quality variations among female and male talkers. Journal of the Acoustical Society of America 87, pp. 820-857. 1990

[15] Strik, H. \& Boves, L. The fundamental frequency subglottal pressure ratio. In Proceedings of EUROSPEECH-89, Vol. 2, pp. 425-428. 1989

[16] Strik, H. \& Boves, L. Control of fundamental frequency, intensity and voice quality in speech. Journal of Phonetics.

[17] Veth, J. de, Cranen, B., Strik, H. \& Boves, L. Extraction of control parameters for the voice source in a text-to-speech system. In Proceedings of ICASSP-90, paper 21.S6a.2. 1990

[18] Kong Jiangping, Study on Phonation Features of Tones in Mandarin, ICPhS2003, Barcelona August 2003 